\title{
Correlation of the HPV genotype with the degree of dysplasia in patients with cervicouter cancer in the General Hospital Naval of high specialty
}

\author{
Oyuki A. Morales ${ }^{1 *}$, Junior J. Araiza ${ }^{1}$, María del R. Garay ${ }^{2}$, \\ Jorge A. Barbabósa ${ }^{2}$, Marlene de la P. Gutiérrez ${ }^{2}$
}

\author{
${ }^{1}$ Department of Gynecology, Postgraduate School in Naval Health, Mexico \\ ${ }^{2}$ Gynecology-Obstetrics Service of the General Hospital Naval of High Specialty (HOSGENAES), Coyoacán \\ Delegation, Mexico
}

Received: 26 September 2017

Received: 03 October 2017

Accepted: 07 October 2017

\section{*Correspondence:}

Dr. Oyuki A. Morales,

E-mail: aricet_512@hotmail.com

Copyright: ( ) the author(s), publisher and licensee Medip Academy. This is an open-access article distributed under the terms of the Creative Commons Attribution Non-Commercial License, which permits unrestricted non-commercial use, distribution, and reproduction in any medium, provided the original work is properly cited.

\begin{abstract}
Background: Cervical-uterine cancer $(\mathrm{CaCu})$ is the second leading cause of death in women worldwide and the first in developing countries. It has been correlated to human papillomavirus (HPV) genotype with cancerous lesions reported in histopathological studies, which tells us about the prognosis of the patients. Objective of present study was to correlate the genotype of HPV to the histopathological result evaluated in these patients at the Naval Hospital of High Specialty.

Methods: A total of 316 women attended at the Naval General High Marine Hospital from 2015 to 2016 with HPV diagnosis were included, the histopathological report was correlated with the HPV genotype determined by the chain reaction of the polymerase (PCR). Statistical tests were applied for Smirnov and Kolmogorov, Chi square, OR, Anova-Kruskal Wallis. Data were processed using the SPSS software version 19 and a $\mathrm{P}<0.05$ was taken as statistical significance.

Results: The mean age was $36.0 \pm 10.2$. The detection of cervical cancer was reported in $3.8 \%$ and stage CIN III $2.5 \%$; the highest proportion of patients were in the CIN I stage (51.3\%), while the CIN II was $9.2 \%$, and up to $30.7 \%$ were classified as HPV infection; only $2.5 \%$ were normal. The types of HPV prevalent by PCR were those at high risk different from 16 and 18 with a rate of $34.8 \%$, then 16 at $5.1 \%$ and 18 at $0.3 \%$. More than half of the cases $(54.4 \%)$ were negative for the serotypes analyzed. In general terms, $\mathrm{CaCu}$ and $\mathrm{CIN}$ III were observed in $15 \%$ of the type 16 , $0 \%$ in type $18,25 \%$ in other high risk and $60 \%$ in negative PCR.

Conclusions: In this study, we obtained essential data that tells us that this population could be treated with low-risk $\mathrm{HPV}$ types associated with $\mathrm{CaCu}$.
\end{abstract}

Keywords: Cervical dysplasia, Human papilloma virus, Polymerase chain reaction, Uterine cervical cancer

\section{INTRODUCTION}

Cervical cancer is the second most common cancer among women worldwide. ${ }^{1}$ It is the second leading cause of mortality due to malignant neoplasia in women, producing up to 11 deaths per day affecting the age group of 25-69 years and only is outweighed by breast cancer. ${ }^{2}$ In Mexico, human papillomavirus infection is the cause of a large percentage of cervical cancer; is one of the main public health problems, with an incidence of $15.5 \%$ and a mortality of $12.8 \% .^{3}$ It has been identified that there are differences in the prevalence of type of HPV. ${ }^{4}$ 
Cervical cancer is caused by oncogenic HPV infection. More than $100 \mathrm{HPV}$ genotypes have been described, of which approximately 40 are responsible for genital infection. ${ }^{5} \mathrm{HPV}$ is classified as low or high risk based on its association with premalignant and malignant lesions, respectively. Low-risk HPVs include 6, 11, 40, 42, 43, $44,54,61,70,72$, and 81 . Of these types, 6 and 11 cause $90 \%$ of cases of anogenital warts and low grade in cervical cells. ${ }^{6,7}$ High risk types include 16, 18, 31, 33, $35,45,52$, and 58, of which 16 and 18 cause approximately $70 \%$ of all cases of invasive cervical cancer. ${ }^{8}$ Some authors have suggested geographical differences in the distribution of HPV can have an impact on the effectiveness of the HPV vaccine in different populations. ${ }^{9}$ In 2005, the International Agency for Research on Cancer reported the worldwide distribution of HPV types in women with cervical cytology normal. The most frequent serotype is HPV 16 followed by HPV 42, 58, 31, 18, 56, 81, 35, 33 and $45 .{ }^{10}$ In Mexico, HPV $16,18,31,33,39,45,53,58$ and 59 have frequently been found in normal cervical samples. ${ }^{11-17}$ In the year 2015 in the United States they report that the most common highrisk human papillomavirus is serotype 16 , associated with precancerous lesions in the cervix and conferring greater risk of disease of high-grade lesions; also reported that $50 \%$ of adenocarcinoma in situ and $50 \%$ of cases of invasive cancer were attributable to HPV infection $18 .^{18}$

\section{METHODS}

The patients with cervical alterations between 2015 and 2016 were 316 with histopathological report, HPV genotyping by polymerase chain reaction, colposcopy surveillance, with cervical cytology; as well as a history and risk factors for $\mathrm{CaCu}$ were reviewed.

According to the results of pathology, the cases were classified according to histological types diagnosed in patients with HPV as follows: normal, changes associated with HPV infection, CIN I, CIN II, CIN III and cervical cancer. From the HPV PCR report the cases of agreement were classified as follows: 16, 18, other high risk and negative. Colposcopies were determined, which were classified as follows: normal, abnormal and $\mathrm{CaCu}$; as to the cytologies were classified as: normal, low grade, high grade and $\mathrm{CaCu}$. The records were searched with a complete medical history and specified risk factors for $\mathrm{CaCu}$ such as age, menarche, onset of active sex life, nulliparity, smoking, sexually transmitted diseases and menopause. Subsequently with the collected data a database was made for statistical analysis. The present study was approved by the Ethics and Local Research Committee, which granted approval with document number 2S.1.798 / JP373 / 16.

\section{Statistical analysis}

The description of the sample was carried out using descriptive statistics with measures of central trend and dispersion, as well as frequency counting, proportions and percentages. For the bivariate and multivariate analysis, inferential statistics were applied, including analysis of variance of one and two factors to compare means and to evaluate the interactions; the Chi square test was applied to compare ratios and Student's t-test to compare two averages of samples with normal distribution. A p equal to or less than 0.05 was considered significant to reject null hypotheses. The data were analyzed with the SPSS package ver 17.0 for Windows, with a significance value of $\mathrm{p}<0.05$.

\section{RESULTS}

The mean age with its respective standard deviation was $36.0 \pm 10.2$ in the range of 17 to 71 years. The average menarche was at $12.6 \pm 1.6$ with 7 years the lowest until 19 the highest. The onset of active sexual life fell in $18.5 \pm 2.9$ years with an interval of 11 to 32 . Only $17.4 \%$ were in the menopause, $42.1 \%$ referred to sexually transmitted diseases, approximately $40 \%$ were positive to alcoholism and $24.1 \%$ to smoking. A little more than half were nulliparous $(50.6 \%)$ and $3.5 \%$ were diagnosed as cervical cancer by colposcopy, but up to $93.4 \%$ were reported with abnormal results; only 5 cases $(1.6 \%)$ were reported as cervical cancer, $8.5 \%$ had high-grade lesions, $66.1 \%$ were low-grade, and $23.7 \%$ were normal.

By the gold standard the identification of cervical cancer reached a rate of $3.8 \%$ and in stage CIN III 2.5\%; (Figure 1) showed that the highest proportion of patients were in the CIN I stage (51.3\%), while the CIN II was $9.2 \%$, and up to $30.7 \%$ were classified as HPV infection, only $2.5 \%$ were normal.

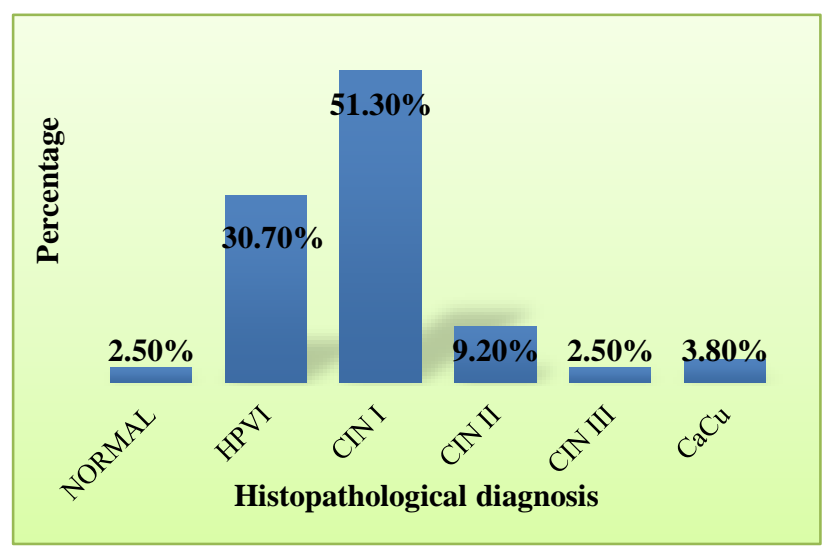

Figure 1: Histopathological diagnoses of cases.

The HPV types prevalent by PCR were those of high grade different to 16 and 18 with a rate of $34.8 \%$, then 16 in $5.1 \%$ and 18 in $0.3 \%$; the combination of 16 plus other high risk were $4.1 \%$ and the combination of 18 plus other high risk were barely $1.3 \%$. In general, type 16 (only or combined) amounted to $9.2 \%, 18$ (only or combined) was present in $1.6 \%$ of the total. It should be noted that more than half of the cases $(54.4 \%)$ were negative for the serotypes analyzed (Figure 2). 


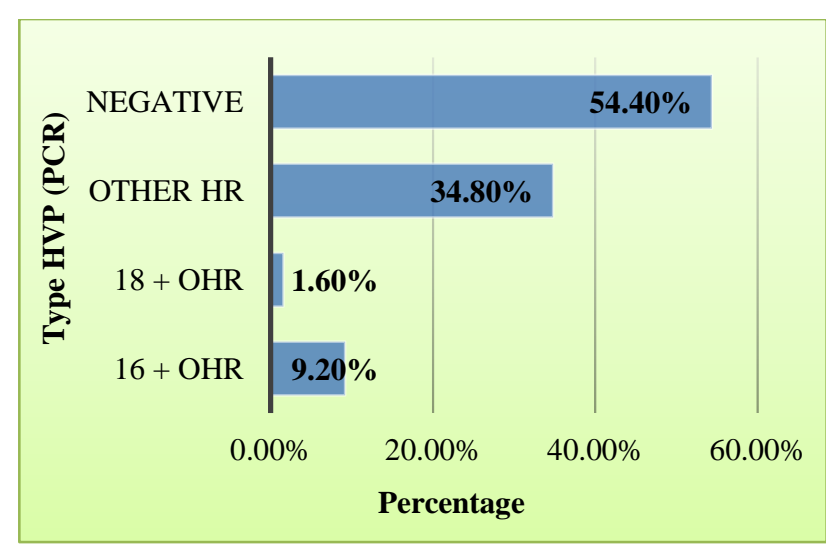

Figure 2: Prevalence of HPV types identified by PCR.

\section{Bivariate and multivariate analysis}

As can be observed (Table 1), the cases of CIN 3-Cacu had a significantly higher mean age than the patients in the other strata of the histopathological diagnosis $(\mathrm{p}=$ 0.0001 according to the analysis of variance of one factor = ANOVA ONE WAY); On the contrary, normal cases started menarche at a significantly higher age than the other diagnostic strata ( $p=0.02$ ANOVA ONE WAY); it is also easy to observe in the same table 1 that up to $65 \%$ of the cases classified as CIN 3-Cacu were in the menopause whereas in the other diagnostic strata the proportions of patients in menopause did not exceed $41 \%$ ( $\mathrm{p}=0.0001$ according to Chi square test). It is also noticeable that, so many cases classified as CIN 1-2 and those with HPVI, had higher proportions of nulliparous patients $(p=0.02)$. Finally, it should be noted that serotype 16 (alone or in combination with other highgrade) was distributed in a significantly higher proportion of cases of CIN 3-Cacu (15\%) versus $12.5 \%$ in normal cases, $9.9 \%$ in CIN1-2 and $6.2 \%$ in the HPVI, note that when the age analysis according to the distribution of serotype 16, the cases of CIN 3-Cacu decreased the age to 33.3 average years, while the cases of IVPH with serotype 16 increased the average age to 43.1 years; serotypes 18 (alone or combined with high grade) were only concentrated in the cases of CIN1-2 and between the normal ones, both with very similar average ages (32.5 years vs 34.0); the other high-grade serotypes were identified mainly in the normal classified cases $(62.5 \%)$ and in the cases CIN 1-2 (37.2\%) but an outstanding fact is that the age of CIN 3-Cacu patients simultaneously carrying serotypes of high levels different to 16 and 18 rises to an average of 52.4 years, that is to say, almost 20 years more than the average of the carriers of the serotype 16 whose age was 33.3 years, also, note that the average age of the cases normal is raised to 43.0 or 13 years longer than normal but carriers of serotypes 16; However, negative cases of serotypes studied were distributed in greater proportion within the diagnoses of HPVI (63.9\%) and within the CIN 3-Cacu (60.0\%), and in both strata the age was higher (39.3 for HPVI vs 47.0 for CIN 3-Cacu) to that of the other two strata or normal and CIN 1-2. $(\mathrm{p}=$ 0.04 when comparing the proportions of the HPV serotype according to the histopathological diagnosis according to the Chi square test).

Table 1: Analysis of sociodemographic factors with histopathological report.

\begin{tabular}{|c|c|c|c|c|c|}
\hline \multirow{3}{*}{ Factors } & \multicolumn{4}{|c|}{ Histopathological diagnosis } & \multirow{3}{*}{ p } \\
\hline & Normal & HPVI & CIN I-II & CIN 3-CaCu & \\
\hline & $(\mathbf{n}=\mathbf{8})$ & $(\mathbf{n}=97)$ & $(n=191)$ & $(\mathrm{n}=\mathbf{2 0})$ & \\
\hline Age & $38.5 \pm 9.2$ & $38.1 \pm 11.0$ & $33.8 \pm 8.3$ & $46.3 \pm 14.4$ & 0.0001 \\
\hline Menarche & $14.0 \pm 2.3$ & $12.3 \pm 1.7$ & $12.6 \pm 1.5$ & $12.9 \pm 1.1$ & 0.02 \\
\hline IVSA & $18.8 \pm 1.8$ & $18.9+7-3.1$ & $18.4 \pm 2.6$ & $18.3 \pm 4.6$ & 0.58 \\
\hline Menopause & $3(37.5 \%)$ & $24(24.7 \%)$ & $15(7.9 \%)$ & $13(65.0 \%)$ & 0.0001 \\
\hline STDs & $3(37.5 \%)$ & $40(41.2 \%)$ & $77(40.3 \%)$ & $13(65.0 \%)$ & 0.19 \\
\hline Nuliparity & $3(37.5 \%)$ & $44(45.4 \%)$ & $108(56.5 \%)$ & $5(25.0 \%)$ & 0.02 \\
\hline \multicolumn{6}{|l|}{ Type of HPV } \\
\hline 16+other HR & $1(12.5 \%)$ & $6(6.2 \%)$ & $19(9.9 \%)$ & $3(15.0 \%)$ & $P$ type of HPV $=0.04$ \\
\hline Age & $29.9 \pm 9.6$ & $43.1 \pm 3.9$ & $32.8 \pm 2.2$ & $33.3 \pm 5.5$ & \\
\hline 18+other HR & $1(12.5 \%)$ & - & $4(2.1 \%)$ & - & \\
\hline Age & $34.0 \pm 9.6$ & - & $32.5 \pm 4.8$ & - & \\
\hline Other HR & $5(62.5 \%)$ & $29(29.9 \%)$ & $71(37.2 \%)$ & $5(25.0 \%)$ & \\
\hline Age & $43.0 \pm 4.2$ & $34.5 \pm 1.7$ & $33.6 \pm 1.1$ & $52.4 \pm 4.2$ & \\
\hline Negative & $1(12.5 \%)$ & $62(63.9 \%)$ & $97(50.8 \%)$ & $12(60.0 \%)$ & \\
\hline Age & $30.0 \pm 9.6$ & $39.3 \pm 1.2$ & $34.1 \pm 0.9$ & $47.0 \pm 2.7$ & \\
\hline P for age & 0.38 & 0.03 & 0.93 & 0.02 & \\
\hline
\end{tabular}

To close the description of Table 1, it should be noted that when comparing the means of age according to serotype within each diagnosis the differences are significant within the HPVI $(\mathrm{p}=0.03)$ and within those of CIN 3-Cacu $(p=0.02)$; this means that the serotypes 
are distributed differently according to the age within each diagnosis, for example, in the HPVI the average ages were: 43.1 for the $16 ; 34.5$ for the other high-grade and 39.3 for the negative cases $(p=0.03)$; in the cases of CIN 3-Cacu the corresponding ages were 33.3 for the 16 ; 52.4 for high grade and 47.0 for negative $(\mathrm{p}=0.02)$ overall and according to the analysis of variance of two factors there is a significant interaction $(p=0.03)$ between the histopathological diagnosis and the serotype for age.

If we analyze the relationship between the STDs strata (SI and NO), note that in the stratum of positive STDs (n $=133$ ) the differences in the proportions of the serotypes are not significant ( $p=0.43 \mathrm{Chi}$ squared) and that in the stratum of negative STDs $(n=183)$ the differences between the proportions of the serotypes according to the histopathological diagnosis are very broad $(p=0.009$ Chi squared). It should be noted that the mean age of the stratum with STD is 35.7 vs 36.3 years of the stratum without STD ( $p=0.61)$ so that the difference between strata is not explained by age. What is distinctive is that, on the one hand, other high-grade serotypes are widely distributed among the diagnoses of both strata and on the other hand it should be noted that serotype 16 is distributed in greater proportion in cases of $\mathrm{CIN} 3-\mathrm{CaCu}$, but of stratum without STDs.

In relation to nulliparity, the differences between the serotypes according to diagnosis are not significant ( $\mathrm{p}=$ $0.33)$ in the nulliparous stratum $(n=160$ but whose average age is 32.1 years versus 40.1 years of the 156 nulliparous ones: $\mathrm{p}=0.0001 \mathrm{t}$ of Student). In the nonnulliparous stratum within normal cases there are no negative serotypes and $80 \%$ correspond to other highgrade serotypes and one case with serotype 18, contrasting within the cases of HPVI, CIN1-2 and CIN 3Cacu Negativity rates exceed $50 \%$ with serotypes 16 in very low proportions $(\mathrm{p}=0.01 \mathrm{Chi}$ square plausibility ratio).

Table 2: Sociodemographic factors related to HPV type.

\begin{tabular}{|lllllll|}
\hline Factors & $\begin{array}{l}\text { Type of HPV } \\
\text { 16 }\end{array}$ & $\mathbf{1 8}$ & OHR & Negative & P \\
\hline Age & $(\mathbf{n = 2 9 )}$ & $(\mathbf{n = 5})$ & $\mathbf{( n = 1 1 0 )}$ & $\mathbf{( n = 1 7 2 )}$ & 0.4 \\
\hline Menarche & $12.8 \pm 10.6$ & $32.8 \pm 7.7$ & $35.1 \pm 9.6$ & $36.9 \pm 10.5$ & $12.4 \pm 1.4$ & 0.41 \\
\hline IVSA & $17.6 \pm 2.1$ & $12.8 \pm 0.8$ & $12.7 \pm 1.6$ & $18.4 \pm 2.9$ & 0.07 \\
\hline Menopause & $5(17.2 \%)$ & 0 & $19.1 \pm 3.1$ & $38(22.1 \%)$ & 0.04 \\
\hline STDs & $15(51.7 \%)$ & $2(40.0 \%)$ & $44(40.0 \%)$ & $72(41.9 \%)$ & 0.72 \\
\hline Alcoholism & $7(24.1 \%)$ & $4(80.0 \%)$ & $46(41.8 \%)$ & $69(41.9 \%)$ & 0.08 \\
\hline Smoking & $6(20.7 \%)$ & $2(40.0 \%)$ & $22(20.0 \%)$ & $46(26.7 \%)$ & 0.47 \\
\hline Nuliparity & $17(58.6 \%)$ & $4(80.0 \%)$ & $59(53.6 \%)$ & $80(46.5 \%)$ & 0.24 \\
\hline
\end{tabular}

Finally, for ethyl alcohol, the differences between the proportions of serotypes according to histopathological diagnosis are only significant ( $p=0.009$ Chi squared) in the stratum of cases with positive alcoholism, whose $n=$ 126 and with a mean age of 34.4 years vs 37.1 years negative cases of alcoholism with $\mathrm{n}=190$ so that age again seems to be a strong confounding factor $(p=0.02$ for the difference of means of age according to Student's t). In normal cases with positive efficacy serotypes 18 and others of high degree were detected and that in the opposite end the cases of $\mathrm{CIN} 3-\mathrm{CaCu}$ within the same stratum of positive alcoholism were only serotypes 16 and the rest were negative serotypes; $(p=0.27$ Chi squared), all the diagnoses shared similar distributions of serotypes 16 and others of high grade (without any case of serotypes 18).

Close the analysis of the data emphasizing that the serotypes were independently related with significant differences in their proportions according to menopause and with significant differences (without being significant) according to age of IVSA and ethylism; (Table 2) that, in the presence of menopause, the rate of negative serotypes is proportionally greater than the proportions of serotype 16 and others of high grade ( $\mathrm{p}=$ $0.04)$.

On the other hand, the IVSA age of patients with other high-grade phenotypes was higher in comparison to those of serotypes 16 and 18 and of negative ones $(p=0.07)$, and, finally, a relevant data is that 4 of 5 cases $(80.0 \%)$ of serotype 18 were identified in patients with positive efficacy whereas within this same stratum of positive efficacy serotype 16 was only identified in $24.1 \%$, other high grade types in $41.8 \%$ and negative serotypes in the $40.1 \%(\mathrm{p}=0.08)$.

\section{Performance of diagnostic methods}

Compared with the gold standard (histopathological diagnosis), colposcopy reveals a sensitivity of $55 \%$ and a specificity of $12.5 \%$, as measured by diagnostic yield as 
reasons for probability to be observed (Table 3) that cases reported as normal by colposcopy remain in the $\mathrm{CaCu}$ area discarded; while those reported as abnormal are only 0.46 times more likely to correspond to $\mathrm{CaCu}$, obviously all cases reported as $\mathrm{CaCu}$ by colposcopy enter the confirmation zone.

In Table 4, the distribution of HPV types by colposcopy and gold standard results are mentioned.
Table 3: Probabilistic comparison of diagnosis by colposcopy and histopathological diagnosis.

\begin{tabular}{|c|c|c|c|}
\hline \multirow[t]{2}{*}{ Colposcopy } & \multicolumn{2}{|c|}{$\begin{array}{l}\text { Histopathological } \\
\text { diagnosis of } \mathrm{CaCu}\end{array}$} & \multirow{2}{*}{$\begin{array}{l}\text { Probability } \\
\text { ratio of } \\
\mathrm{CaCu}\end{array}$} \\
\hline & Yes & No & \\
\hline Normal & 0 & $10(3.4 \%)$ & Discarded \\
\hline Abnormal & $9(45.0 \%)$ & $286(96.6 \%)$ & 0.46 \\
\hline CACU & $11(55.0 \%)$ & 0 & Confirmed \\
\hline Total & 20 & 296 & \\
\hline
\end{tabular}

Table 4: Distribution of HPV types according to colposcopy and presence or absence of $\mathrm{CaCu}$.

\begin{tabular}{|c|c|c|c|c|c|}
\hline \multirow{2}{*}{ Colposcopy } & & & \multicolumn{2}{|l|}{$\mathrm{CaCu}$} & \multirow{2}{*}{ Total } \\
\hline & & & With & Without & \\
\hline \multirow{4}{*}{ Normal } & \multirow{3}{*}{$\begin{array}{l}\text { Type HPV } \\
\text { (PCR) }\end{array}$} & 16 +other HR & & $1(10 \%)$ & $1(10 \%)$ \\
\hline & & Other HR & & $2(20 \%)$ & $2(20 \%)$ \\
\hline & & Negativos & & $7(70 \%)$ & $7(70 \%)$ \\
\hline & Total & & & $10(100 \%)$ & $10(100 \%)$ \\
\hline \multirow{5}{*}{ Abnormal } & \multirow{4}{*}{$\begin{array}{l}\text { Type HPV } \\
\text { (PCR) }\end{array}$} & 16 +other HR & $1(11.1 \%)$ & $25(8.7 \%)$ & $26(8.8 \%)$ \\
\hline & & 18+other HR & $0(0 \%)$ & $5(1.7 \%)$ & $5(1.7 \%)$ \\
\hline & & Other HR & $3(33.3 \%)$ & $103(36 \%)$ & $106(35.9 \%)$ \\
\hline & & Negative & $5(55.6 \%)$ & $153(53.5 \%)$ & $158(53.6 \%)$ \\
\hline & Total & & $9(100 \%)$ & $286(100 \%)$ & $295(100 \%)$ \\
\hline \multirow{4}{*}{$\mathrm{CaCu}$} & \multirow{3}{*}{$\begin{array}{l}\text { Type HPV } \\
\text { (PCR) }\end{array}$} & 16 +other HR & $2(18.2 \%)$ & & $2(18.2 \%)$ \\
\hline & & Other HR & $2(18.2 \%)$ & & $2(18.2 \%)$ \\
\hline & & Negative & $7(63.6 \%)$ & & $7(63.6 \%)$ \\
\hline & Total & & $11(100 \%)$ & & $11(100 \%)$ \\
\hline
\end{tabular}

For cytology the sensitivity is $25.0 \%$ with $20 \%$ specificity; however, measured the diagnostic yield as a likelihood ratio see (Table 5) that a normal cytology report equals a probability of 0.83 that corresponds to $\mathrm{CaCu}$, if it is reported low-grade the probability is 0.07 times that it is $\mathrm{CaCu}$, but if it is reported as high-grade it is 8.70 times more likely to correspond to $\mathrm{CaCu}$.

Table 5: Probabilistic comparison of: diagnosis by cervical cytology and histopathological diagnosis.

\begin{tabular}{|c|c|c|c|}
\hline \multirow[t]{2}{*}{ Cytology } & \multicolumn{2}{|c|}{$\begin{array}{l}\text { Histopathological } \\
\text { Diagnosis of } \mathrm{CaCu}\end{array}$} & \multirow{2}{*}{$\begin{array}{l}\text { Probability } \\
\text { ratio of } \\
\mathrm{CaCu}\end{array}$} \\
\hline & Yes & No & \\
\hline Normal & $4(20.0 \%)$ & $71(24.0 \%)$ & 0.83 \\
\hline Low grade & $1(5.0 \%)$ & $208(70.3 \%)$ & 0.07 \\
\hline High grade & $10(50.0 \%)$ & $17(5.7 \%)$ & 8.7 \\
\hline $\mathrm{CaCu}$ & $5(25.0 \%)$ & 0 & Confirmed \\
\hline Total & 20 & 296 & \\
\hline
\end{tabular}

\section{DISCUSSION}

The incidence of cervical cancer in young women continues to increase; in this study with a mean age for $\mathrm{CaCu}$ of $46.3 \pm 14.4$; very similar to that reported in the United States with a mean age at diagnosis of 48 years. ${ }^{19}$ In Mexico there is an incidence of $15.5 \%$ and a mortality of $12.8 \% .^{3} \mathrm{~A}$ detection rate of $3.8 \%$ for $\mathrm{CaCu}$ and $2.5 \%$ for CIN III has been reported in this study. A low ratio of $\mathrm{CaCu}$ with HPV 16 and 18 was found to be different from what is currently reported in the literature and different population; in general the $\mathrm{CaCu}$ and $\mathrm{CIN}$ III were observed $15 \%$ in type $16,0 \%$ in type $18 ; 25 \%$ in others at high risk and $60 \%$ in negative PCR reports, which makes us think that they could be low risk HPV; which differs to studies where it is mentioned that up to $70 \%$ of cases of $\mathrm{CaCu}$ are mainly caused by types 16 and 18.

Of the patients diagnosed with $\mathrm{CaCu}(\mathrm{n}=20), 65 \%$ (13) reported having undergone sexually transmitted diseases, which coincides with that reported in the literature, where STDs are considered a risk factor for $\mathrm{CaCu}$.

The average age for initiation of active sexual life in cancer patients was $18.3 \pm 4.6$; it is worth remembering that it is considered a risk factor if the first sexual relation is 21 years; the risk is approximately 1.5 times in 18 to 20 years and double for those under 18 years of age. ${ }^{20}$ Although smoking is considered a risk factor for presenting $\mathrm{CaCu}$; there was no statistical significance in relation to it, as in other literature where smoking is not associated with a significantly higher risk of cervical cancer compared to nonsmokers. ${ }^{20-22}$ 
In the studied population, $3.5 \%$ were diagnosed as cervical cancer by colposcopy, but up to $93.4 \%$ were reported with abnormal results; so that in this analysis we found a sensitivity of $55 \%$ and specificity of $12.5 \%$ in comparison with the gold standard (histopathology); it should be mentioned that colposcopy using the criteria of the International Federation of Cervical Pathology and Colposcopy of 2011 reports a sensitivity of 86 percent and a specificity of 30 percent, which differs in our study. Only 5 cases $(1.6 \%)$ were reported as cervical cancer, $8.5 \%$ with high grade lesions, $66.1 \%$ low grade and $23.7 \%$ normal, with a sensitivity of $25 \%$ and specificity of $20 \%$; In a systematic review, sensitivity and specificity varied significantly, sensitivity ranged from $30 \%$ to $87 \%$ and specificity ranged from $86 \%$ to $100 \%$, for which it is mentioned that it is a dependent operator study; even so in this analysis, these values are reported below expectations..$^{23,24}$

\section{CONCLUSION}

Cervical cancer continues to be one of the pathologies of higher morbidity and mortality in the female population. In our population with detection of invasive $\mathrm{CaCu}$ of $3.8 \%$ and cancer in situ of $2.5 \%$; despite the advances in screening, treatment and follow-up, it has not been possible to modify the natural course of the disease. The mean age of patients with cervical cancer was 46 years, ranging from 17 to 71 years; so, it is recommended that patients with risk factors should be considered for a broader follow-up, thus avoiding diseases of advanced stages, their complications and decreasing the quality of life of patients.

Compared to the gold standard (histopathological), cytology presented a sensitivity of $25 \%$ and specificity of $20 \%$; colposcopy had a sensitivity of $55 \%$ and specificity of $12.5 \%$; so, it is recommended to perform more than one screnning study for further detection.

In this study we are reporting some interesting data on the risk factors and the presence of HPV genotypes with respect to the histopathology report present in our study population; which could be very useful to give an earlier detection of the disease and to be able to carry out the ideal treatment for each patient, with less complications; in this study we can conclude that the knowledge of HPV genotype as well as risk factors are data that could be applied in clinical practice in order to give an overview of the pathology, thus reducing institutional costs by giving preventive management and even timely treatment.

Concluding that, in our population, patients who were diagnosed with cervical cancer or cervical dysplasia, reported a negative PCR for high-risk HPV; which would make us think of including a broader PCR in which low risk HPV is detected and more specific for high risk HPV; which could be present in our population.

Funding: No funding sources
Conflict of interest: None declared

Ethical approval: The study was approved by the Institutional Ethics Committee

\section{REFERENCES}

1. Ferlay J, Bray F, Pisani P, Parkin DM. Globocan 2002 cancer incidence. Mortality and prevalence worldwide. IARC Cancer Base no 5, version 2.0, IARC Press, Lyon, France;2004.

2. Lazcano-Ponce EC, Rascón-Pacheco RA, LozanoAscencio R, Velasco-Mondragón HE. Mortality from cervical carcinoma in Mexico: impact of screening, 1980-1990. Acta Cytologica. 1996;40(3):506-12.

3. Joseph Monsonego, J. Thomas Cox, Catherine Behrens, Maria Sandri, Eduardo L. Franco Poh-Sin Yap, Warner Huh. Prevalence of high risk human papilloma virus genotypes and associated risk of cervical precancerous lesions in large U.S. screening population: data from the ATHENA trial. Gynecol Oncol. 2015;137:47-54.

4. Salcedo M, Pina-Sanchez P, Vallejo-Ruiz V, Monroy-Garcia A, Aguilar-Lemarroy A, CortesGutierrez EI, et al. Human papillo-mavirus genotypes among females in Mexico: a study from the Mexican Institute for Social Security. Asian Pac J Cancer Prev. 2014;15(23):10061-6.

5. De Villiers EM, Fauquet C, Broker TR, Bernard HU, zur Hausen H. Classification of papillomaviruses. Virology. 2004 Jun 20;324(1):17-27.

6. Stanley M. Prophylactic HPV vaccines: prospects for eliminating ano-genital cancer. Br J Cancer. 2007 May 1;96(9):1320.

7. Muñoz N, Bosch FX, de Sanjosé S, Herrero R, Castellsagué X, Shah KV et al. Epidemiologic classification of human papillomavirus types associated with cervical cancer. N Engl J Med. 2003 Feb 6;2003(348):518-27.

8. Smith JS, Lindsay L, Hoots B, Keys J, Franceschi S, Winer $R$ et al. Human papillomavirus type distribution in invasive cervical cancer and highgrade cervical lesions: a meta- analysis update. Int $\mathbf{J}$ Cancer. 2007 Aug 1;121(3):621-32.

9. Mammas IN, Vageli D, Spandidos DA. Geographic variations of human papilloma virus infection and their possible impact on the effectiveness of the vaccination programme. Oncology Reports. 2008 Jul $1 ; 20(1): 141-5$.

10. Clifford GM, Gallus S, Herrero R, Munoz N, Snijders PJ, Vaccarella $S$ et al. Worldwide distribution of human papillomavirus types in cytologically normal women in the International Agency for Research on Cancer HPV prevalence surveys: a pooled analysis. Lancet. 2005 Sep 23;366(9490):991-8.

11. Orozco-Colín A, Carrillo-García A, Méndez-Tenorio A, Ponce-de-León S, Mohar A, MaldonadoRodríguez $\mathrm{R}$ et al. Geographical variation in human papillomavirus prevalence in Mexican women with 
normal cytology. Int $\mathbf{J}$ Infect Dis. 2010 Dec 31;14(12):e1082-7.

12. Illades-Aguiar B, del Carmen Alarcón-Romero L, Antonio-Véjar V, Zamudio-López N, Sales-Linares N, Flores-Alfaro E et al. Prevalence and distribution of human papillomavirus types in cervical cancer, squamous intraepithelial lesions, and with no intraepithelial lesions in women from Southern Mexico. Gynecol Oncol. 2010 May 31;117(2):291-6.

13. Velázquez-Márquez N, Paredes-Tello MA, PérezTerrón H, Santos-López G, Reyes-Leyva J, VallejoRuiz V. Prevalence of human papillomavirus genotypes in women from a rural region of Puebla, Mexico. Int J Infect Dis. 2009 Nov 30;13(6):690-5.

14. Illades-Aguiar B, Cortés-Malagón EM, AntonioVéjar V, Zamudio-López N, del Carmen AlarcónRomero L, Fernández-Tilapa G, Hernández-Sotelo D, Terán-Porcayo MA, Flores-Alfaro E, LeyvaVázquez MA. Cervical carcinoma in Southern Mexico: Human papillomavirus and cofactors. Cancer Detect Prevent. 2009 Dec 31;32(4):300-7.

15. Sánchez-Anguiano LF, Alvarado-Esquivel C, ReyesRomero MA, Carrera-Rodríguez M. Human papillomavirus infections in women seeking cervical Papanicolaou cytology of Durango, Mexico: prevalence and genotypes. BMC Infect Dis. 2006 Feb 20;6(1):27.

16. Lazcano- Ponce E, Herrero R, MUnoz N, Cruz A, Shah KV, Alonso P, HERNandez P, SALMERon J, HERNandez M. Epidemiology of HPV infection among Mexican women with normal cervical cytology. Int J Cancer. 2001 Feb 1;91(3):412-20.

17. Torroella-Kouri M, Morsberger S, Carrillo A, Mohar A, Meneses A, Ibarra M, Daniel RW, Ghaffari AM, Solorza G, Shah KV. HPV prevalence among Mexican women with neoplastic and normal cervixes. Gynecologic Oncol. 1998 Jul 31;70(1):11520.

18. Peralta-Rodríguez R, Romero-Morelos P, VillegasRuíz V, Mendoza-Rodríguez M, Taniguchi-Ponciano K, González-Yebra B, Marrero-Rodríguez D, Salcedo M. Prevalence of human papillomavirus in the cervical epithelium of Mexican women: metaanalysis. Infect Agents Cancer. 2012 Dec 3;7(1):34.
19. Ries LAG, Melbert D, Krapcho M. SEER Cancer Statistics Review, 1975-2004. Instituto Nacional del Cáncer; Bethesda, MD;2007.

20. International Collaboration of Epidemiological Studies of Cervical Cancer. Comparison of risk factors for invasive squamous cell carcinoma and adenocarcinoma of the cervix: collaborative reanalysis of individual data on 8,097 women with squamous cell carcinoma and 1,374 women with adenocarcinoma from 12 epidemiological studies. Int J Cancer. 2007; 120:885.

21. Castle PE, Wacholder S, Lorincz AT, Scott DR, Sherman ME, Glass AG et al. A prospective study of high-grade cervical neoplasia risk among human papillomavirus-infected women. J National Cancer Institute. 2002 Sep 18;94(18):1406-14.

22. International Collaboration of Epidemiological Studies of Cervical Cancer, Appleby P, Beral V, et al. Carcinoma of the cervix and tobacco smoking: collaborative reanalysis of individual data on 13,541 women with carcinoma of the cervix and 23,017 women without carcinoma of the cervix from 23 epidemiological studies. Int J Cancer. 2006;118:1481.

23. Bornstein J, Bentley J, Bösze P, Girardi F, Haefner $\mathrm{H}$, Menton $\mathrm{M}$ et al. 2011 colposcopic terminology of the International Federation for Cervical Pathology and Colposcopy. Obstet Gynecol. 2012 Jul 1;120(1):166-72.

24. Nanda K, McCrory DC, Myers ER, Bastian LA, Hasselblad V, Hickey JD, Matchar DB. Accuracy of the papanicolaou test in screening for and follow-up of cervical cytologic abnormalitiesa systematic review. Annals Internal Med. 2000 May 16;132(10):810-9.

Cite this article as: Morales OA, Araiza JJ, del RGM, Barbabósa JA, de la PGM. Correlation of the HPV genotype with the degree of dysplasia in patients with cervicouter cancer in the General Hospital Naval of high specialty. Int J Reprod Contracept Obstet Gynecol 2017;6:4814-20. 